\title{
Historiographie du Parti communiste canadien 1960-1982
}

\section{Bernard Dionne}

Volume 37, numéro 2, septembre 1983

Travailleurs et mouvements sociaux

URI : https://id.erudit.org/iderudit/304159ar

DOI : https://doi.org/10.7202/304159ar

Aller au sommaire du numéro

Éditeur(s)

Institut d'histoire de l'Amérique française

ISSN

0035-2357 (imprimé)

1492-1383 (numérique)

Découvrir la revue

Citer cette note

Dionne, B. (1983). Historiographie du Parti communiste canadien 1960-1982. Revue d'histoire de l'Amérique française, 37(2), 309-319.

https://doi.org/10.7202/304159ar d'utilisation que vous pouvez consulter en ligne.

https://apropos.erudit.org/fr/usagers/politique-dutilisation/ 
NOTE CRITIQUE

\title{
HISTORIOGRAPHIE DU PARTI COMMUNISTE CANADIEN 1960-1982
}

\author{
BERNARD DIONNE \\ Professeur d'histoire \\ CEGEP de Saint-Jérôme
}

Depuis la fin des années soixante, la production d'ouvrages historiques sur la classe ouvrière canadienne s'est accrue à un rythme rapide. La conjoncture sociale et politique qui va de la «révolution tranquille» d'hier à la «tranquille réaction» d'aujourd'hui y est sans doute pour quelque chose. Ajoutons à cela la crise de société que connaissent les pays occidentaux et les pays dits socialistes - dont la récente interdiction d'un syndicat de dix millions de membres par un parti «ouvrier» au pouvoir n'en est que la plus récente manifestation et nous avons dessiné à grands traits le contexte dans lequel plusieurs ouvrages sur le Parti communiste canadien (PCC) et même une toute récente histoire «officielle» ont été réalisés. L'heure est donc au bilan de cette production historiographique. Nous nous en tiendrons ici aux ouvrages publiés au Canada depuis 1960 portant sur l'histoire globale du PCC ou sur son histoire au Québec ${ }^{1}$. Un thème majeur a retenu notre attention: le Parti communiste comme «avant-garde de la classe ouvrière» et les causes de son échec dans l'accomplissement de sa mission historique qui est de faire la révolution.

\section{Le Parti communiste, parti d'avant-garde de la classe ouvrière}

Comme le soulignait fort justement l'historien anglais Eric Hobsbawm, «chaque PC a été le fruit du mariage de deux partenaires mal assortis: une gauche nationale et la Révolution d'Octobre» ${ }^{2}$. En créant le PC canadien, les communistes eurent l'impression de donner à la classe ouvrière l'outil de direction de ses combats qui lui avait fait défaut au cours de la Première Guerre mondiale et au cours de la grève générale de Winnipeg en 1919. C'est le point de vue qu'exprime l'histoire officielle du PCC, Canada's Party of Socialism, History of the

\footnotetext{
1 Il s'agit ici du texte légèrement remanié d'une communication présentée au congrès de l'IHAF les 21-22 octobre 1982. Nous avons mis de côté l'historiographie de la question nationale québécoise car cela faisait l'objet d'une autre communication. Merci à Gérard Bouchard pour ses critiques et suggestions.

Eric Hobsbawm, Revolutionaries (New York, Pantheon Books, 1973) (Notre traduction).
}

RHAF, vol. 37, no 2, septembre 1983 
Communist Party of Canada, 1921-1976 ${ }^{3}$. Décrivant le contexte du début du siècle, les auteurs - une commission d'histoire du PCC comprenant le secrétaire général du Parti, William Kashtan et plusieurs membres de la direction - parlent toujours de la classe ouvrière canadienne, de son enthousiasme pour la révolution russe, de sa mobilisation contre la guerre mondiale, de l'accueil chaleureux qu'elle aurait réservé aux idées de Lénine, et ainsi de suite. Selon eux, la classe ouvrière était mûre pour un parti ouvrier révolutionnaire de type nouveau, marxiste-léniniste, avec les caractéristiques qu'on lui connaît, telles que codifiées dans les célèbres vingt et une conditions d'admission à l'Internationale communiste. Que ce parti n'ait jamais réussi à s'enraciner durablement dans cette classe ouvrière censée l'attendre impatiemment n'étonne pas les auteurs de Canadian Party of Socialism.

L'ancien communiste français Pierre Daix disait «qu'aujourd'hui, un historien communiste, prisonnier de la généalogie et des héritages, ne peut être que commémoratif ${ }^{4}$. Effectivement, l'histoire du PCC commémore les bons coups du Parti, surtout ceux des années trente: la création de la Ligue d'Unité ouvrière avec ses 40000 membres que personne n'a jamais vraiment recensés; le procès de Tim Buck, le chef du Parti, et la grande assemblée de 20000 personnes qui l'ont accueilli à sa sortie de prison au Maple Leaf Garden de Toronto; la glorieuse épopée des 1200 membres du bataillon Mackenzie-Papineau des brigades internationales à la guerre d'Espagne; le docteur Norman Bethune en Chine, etc...

Pour les militants, c'est sans doute un ouvrage réconfortant, utile, nécessaire même. Pour ceux et celles qui cherchent à connaitre et à comprendre l'histoire de la classe ouvrière canadienne, par contre, c'est un document qui déçoit. En fait, par sa méthode d'analyse qui consiste à légitimer la direction du Parti, à condamner les dissidents avec la bonne vieille méthode de la reconstruction historique a posteriori des biographies de militants, celles de Michael Buhay et de J.B. Salsberg notamment, et à escamoter les questions gênantes telle que l'attitude du Parti devant le pacte germano-soviétique d'août 1939, cet ouvrage pose plus de problèmes qu'il n'en résout, à notre avis.

Toutefois, l'intérêt même d'une histoire «officielle» du PCC ne devrait pas nous échapper: il est toujours éclairant de suivre les méandres du discours historique communiste, pourvu qu'on en fasse une lecture critique et qu'on parvienne à situer cette production dans son contexte, dans les débats de ligne qui ont certainement marqué la rédaction de chacun des chapitres.

\footnotetext{
3 Canada's Party of Socialism, History of the Communist Party of Canada, 1921-1976 (Toronto, Progress Books, 1982), 319 p.

4 Pierre Daix, «L'Histoire à l'école du Parti», H. Histoire, 1 (mars 1979): 154.
} 
À côté de cette histoire officielle, on retrouve présentement quatre grands courants critiques de l'histoire du PCC au Canada. Il s'agit des courants que l'on pourrait sommairement identifier comme suit: réactionnaire, exogène, révisionniste et néo-léniniste.

1- Le courant «réactionnaire» ne sera pas analysé ici: nous tenions à signaler l'existence d'une historiographie bourgeoise qui, depuis les débuts du PCC, s'acharne à en déformer les objectifs et l'histoire pour mieux le discréditer. Ce courant s'est particulièrement affirmé au cours des années cinquante 5 au plus fort de la guerre froide, puis, après le déclin du PCC, il s'est contenté d'en souligner la disparition avec une joie non dissimulée.

2- Le courant «exogène» veut quant à lui montrer l' «étrangeté» du PCC au Canada. Pour ce courant, en effet, le PCC est le produit d'une greffe difficile sur un corps récalcitrant, la classe ouvrière canadienne, dans un contexte résolument hostile. Ivan Avakumovic cherche dans The Communist Party In Canada - A History à prouver que les succès du PCC n'étaient dus qu'à son implantation dans des communautés ethniques où, dit-il, «leurs membres avaient une vie différente de celle de la plupart des Canadiens» ${ }^{6}$.

Une prédisposition à accepter des explications et des solutions simplistes, une facilité à mettre en pratique des préceptes organisationnels empruntés à l'étranger et largement inapplicables dans le système politique canadien ont pu créer une sous-culture communiste dans quelques parties du Canada. ${ }^{7}$

L'autoritarisme au plan organisationnel, les nombreux changements inexpliqués dans l'orientation du Parti (dont l'exemple classique restera le passage brutal de l'antifascisme des années trente à la politique du pacte germano-soviétique de août 1939 à juin 1941), l'identification totale du PCC à l'URSS et son «jargon marxiste» inapproprié en Amérique du Nord expliqueraient l'échec du Parti selon Avakumovic. Sa composition ethnique, son incapacité à créer des racines profondes dans le mouvement syndical canadien et dans l'intelligentsia ont réduit le Parti à ce que Salsberg, l'organisateur syndical du PCC, appelait «un parti de groupes restreints et de zones électorales spécifiques, de petits îlots sur la grande scène nationale» ${ }^{8}$.

Pour William Rodney, auteur d'une étude très fine sur le Parti des années 1919 à 1929, «la faillite du PCC à mobiliser les masses canadiennes doit être attribuée à l'idéologie marxiste qui, basée sur les rap-

\footnotetext{
5 À titre d'exemple, signalons: Gérard Dion, «Les communistes et les unions ouvrières», Relations industrielles, 11,4 (sept. 1956): 234-253.

6 Ivan Avakumovic, The Communist Party in Canada, A History (Toronto, McLelland and Stewart, 1974), $309 \mathrm{p}$.

7 Ibid., 275 (notre traduction).

$8 \quad$ Ibid., 278 (notre traduction).
} 
ports de classe, s'est avérée un instrument désuet et inefficace» ${ }^{9}$. Rodney s'attache à démontrer que la lutte pour le pouvoir qui élimina Maurice Spector et Jack MacDonald de leur poste de dirigeant en 1929 au profit de l'équipe de Tim Buck, Leslie Morris et Stewart Smith, de même que la prise du pouvoir total par Staline en URSS et dans le Komintern, discréditèrent le Parti et contribuèrent à l'isoler au sein de la gauche canadienne.

Ce thème du caractère exogène du Parti est aussi largement développé dans l'abondante littérature canadienne-anglaise sur la Cooperative Commonwealth Federation (CCF) et sur le Nouveau Parti Démocratique (NPD). Dans son étude sur la $C_{C} \mathrm{~F}^{10}$, Walter Young a présenté le PCC comme une force politique qui n'a cherché qu'à concurrencer la $\mathrm{CCF}$ et à diviser le mouvement ouvrier canadien; Gad Horowitz, quant à lui, ne parle des communistes dans son ouvrage sur la politique et le mouvement ouvrier au Canada ${ }^{11}$ que pour montrer leur incapacité congénitale à s'adapter à la démocratie canadienne; enfin, Terry Copp a cherché à camoufler l'ampleur de l'implantation syndicale du PCC dans son livre sur les syndicats de l'industrie électrique au Canada ${ }^{12}$. Le travail qu'il a réalisé avec Desmond Morton sur le mouvement ouvrier canadien ${ }^{13}$ présente d'ailleurs la CCF et le NPD comme les seuls produits authentiques du mouvement ouvrier face à un PCC contrôlé par Moscou.

3- Il y a par ailleurs un courant que nous pourrions nommer «révisionniste», composé d'anciens militants ou de critiques sympathiques à leur objet sans être d'accord avec l'ensemble de la ligne politique du PCC. Ainsi, Norman Penner, dans The Canadian Left, A Critical Analysis $^{14}$, reprend la perspective de Hobsbawm à laquelle nous faisions allusion précédemment. En effet, selon Penner, «le PCC a émergé du mouvement socialiste qui grandissait au Canada depuis $1890 \gg^{15}$.

Voyant dans le succès de la révolution russe un encouragement profond, les communistes canadiens ont choisi librement de s'affilier à la III' Internationale et d'en accepter les vingt et une conditions d'adhésion. Ici, pas question de "greffe» mais plutôt de tentative d'union libre, pourrait-on dire, qui échoua à cause de la domination d'un des

\footnotetext{
$9 \quad$ William Rodney, Soldiers of the International. A History of the Communist Party of Canada, 1919-1929 (Toronto, University of Toronto Press, 1968), p.v. (notre traduction).

${ }_{10}$ Walter Young, The Anatomy of a Party: The National C.C.F. (Toronto, University of Toronto Press, 1969).

11 Gad Horowitz, Canadian Labour in Politics (Toronto, University of Toronto Press, 1968).

12 Terry Copp, Le S.I.T.E. au Canada (Kitchener, Cumnock Press, 1979).

13 Desmond Morton et Terry Copp, Working People, An Illustrated History of Canadian Labour (Ottawa, Deneau Publishers, 1980).

14 Norman Penner, The Canadian Left: A Critical Analysis (Scarborough, Prentice-Hall, 1977) 287 p. (notre traduction)

15 Ibid., 78.
} 
partenaires: la dépendance du PCC envers l'Internationale communiste (IC) a nui à l'image du PCC qui, autrement, aurait pu selon Penner rallier une plus grande partie de la gauche canadienne.

Penner a bien noté ce phénomène de l'écart entre la théorie de l'IC et la pratique révolutionnaire au Canada. Le PCC a tenté de les concilier lors des batailles réformistes quotidiennes. Sur ce terrain, le PCC était même souvent à la droite de la $\mathrm{CCF}$, phénomène que nous avons observé en étudiant le PCC pendant la Deuxième Guerre mondiale ${ }^{16}$. Robert Comeau et moi-même avons pu constater dans Les communistes au Québec, $1936-1956^{17}$ qu'après la guerre, le PCC a suivi la ligne de compromis historique avant la lettre que Staline avait imposée aux Partis communistes, allant même jusqu'à dissoudre la III ${ }^{\mathrm{e}}$ Internationale pour plaire aux Alliés en 1943: le PC a changé de nom et adopté un programme réformiste et une ligne politique nationaliste, tentant bien tardivement de prendre de vitesse une CCF mieux implantée et occupant déjà le terrain de la social-démocratie.

Mais en agissant de la sorte, le Parti Ouvrier-Progressiste (POP, nouveau nom du PCC après 1943) ne se démarquait plus de la CCF que par un attachement permanent à l'URSS: il ne parvenait pas à trouver la place qui aurait pu lui revenir sur la scène politique canadienne, encore moins sur la scène politique québécoise, d'autant plus que les préceptes léninistes qui constituaient sa doctrine en matière d'organisation et le caractère subversif et semi-clandestin qui en découlait maintenaient le Parti en marge de la vie politique canadienne.

Les partisans de ce courant «critique» recherchent donc à l'intérieur de la doctrine léniniste les bases théoriques et organisationnelles de l'échec du Parti dans le contexte canadien où la tradition démocratique, l'existence de grands syndicats et d'un influent Parti socialdémocrate auraient dû interdire toute tentative d'application mécanique d'une théorie élaborée dans un tout autre contexte. Mais à la différence des courants réactionnaire et «exogène», les «révisionnistes» considèrent que le Parti a représenté une fraction réelle de la gauche canadienne et a donné une voix originale à des milliers de sans-emplois, d'immigrants et d'ouvriers à la recherche d'une société meilleure.

4- Mais il faut tenir compte d'une autre tendance qui s'est particulièrement développée au cours des années soixante-dix et qui est liée à l'émergence du mouvement marxiste-léniniste et à la résurgence du trotskysme au Canada. Nous voulons parler de ceux qui cherchent à tout prix à démontrer la «trahison» du PC afin de justifier leur propre

\footnotetext{
16 Bernard Dionne, La politique du Parti communiste canadien au cours de la Seconde Guerre mondiale, Thèse M.A. (Histoire), (UQAM, 1979), 297 p.

17 Robert Comeau et Bernard Dionne, Les communistes au Québec, 1936-1956 (Montréal, Les Presses de l'Unité, 1980). On pourra y consulter une bibliographie plus complète sur le PCC au Québec et au Canada.
} 
projet politique et leur propre existence en tant qu'avant-garde de la classe ouvrière.

On peut trouver un exemple parfait de cette tendance dans l'oeuvre de Férgus McKean, ancien dirigeant du Parti en Colombie britannique qui fut expulsé en 1945 et qui accusa le Parti d'avoir trahi la cause révolutionnaire dès 1935 , lorsqu'il avait suivi la ligne des «fronts populaires». Son texte ${ }^{18}$, vite oublié, sera retrouvé et publié par le Parti communiste du Canada (marxiste-léniniste) (PCC (m-1) puis réédité par le groupe En lutte!, l'un et l'autre y trouvant matière à alimenter leur postulat de la trahison du Parti. En fait d'arguments on y trouve beaucoup de citations de Marx, Engels, Lénine, Staline, Dimitrov et Earl Browder, dirigeant du PC aux États-Unis. Avec la revue Mobilisation $^{19}$, qui publia en 1974 une soi-disant étude historique sur le PCC cousue du «fil rouge» dénonçant l'opportunisme, l'économisme et le révisionnisme, les débats s'envenimèrent: le PC avait-il trahi en 1935, en 1943 ou en 1956 lorsqu'il approuva le rapport Krouchtchev contre les crimes de Staline?

Finalement, Ian Angus, partant du point de vue trotskyste, a voulu montrer avec son livre Canadian Bolsheviks ${ }^{20}$, publié en 1982, que le PCC a été rien de moins que l'enjeu d'un coup d'État ourdi par une minorité corrompue et bourgeoise. Voilà comment il explique la prise du pouvoir par Tim Buck qui arriva à la tête du Parti en 1929 après s'être débarrassé du trotskyste Maurice Spector et de l'ex-secrétaire général Jack MacDonald. A partir de ce moment, selon Angus, le PCC devint ultra-gauchiste, à l'image de l'Internationale communiste d'ailleurs et il se coupa ainsi de toute audience réelle dans la classe ouvrière canadienne, permettant ainsi à la CCF de prendre le leadership de la gauche en 1933.

D'autres chercheurs, d'obédience trotskyste, ont montré paradoxalement que c'est plutôt la poursuite d'une ligne trop collaborationniste qui aurait entraîné la déchéance du $\mathrm{PC}^{21}$. Bref, l'histoire du PC vient ici à la rescousse de ceux qui cherchent une réponse à la fameuse question: que serait-il arrivé si? Si MacDonald était resté à la tête du Parti? Si c'est Trotsky qui avait fait assassiner Staline? Si le PC avait créé une vraie centrale syndicale avec la Ligue d'Unité ouvrière, une sorte de CGT canadienne? Derrière cette tentative de réécrire l'histoire, il y a cette idée fondamentale que la classe ouvrière est révolutionnaire

\footnotetext{
18 Fergus McKean, Communism versus Opportunism (Vancouver, the Organizing Committee, 1946); réédition en fac-similé par les Presses de l'Unité (Montréal, 1980), 324 p.

19 «Perspectives historiques sur le Parti communiste du Canada», Mobilisation, 4,4 (1974): $1-30$.

20 Ian Angus, Canadian Bolsheviks, The Early Years of the Communist Party of Canada (Montréal, Vanguard Publications, 1981), 404 p.

21 Magnus Isacsson, Les perspectives d'alliance du Parti communiste canadien au Québec (1935-1939), communication au congrès de l'IHAF (octobre 1975), ronéo, 22 p.
} 
en soi et que c'est la faute aux dirigeants si la révolution n'a pas éclaté au Canada. Nous croyons qu'au lieu de perdre son temps à se demander si le PC a «trahi» quoi que ce soit, il vaut mieux se demander s'il a réussi ou non, s'il pouvait réussir ou non à représenter une tendance significative dans le mouvement ouvrier canadien. Et à cette question, il n'y a pas de réponse toute simple: la crise et la guerre ont révélé les immenses capacités des communistes à mettre en place des syndicats - Irving Abella leur reconnaît un rôle majeur dans l'établissement du Congress for Industrial Organization (CIO) au Canada ${ }^{22}$ - à soulever les questions politiques majeures parmi les travailleurs, à organiser un vaste courant d'opinion pour un effort de guerre démocratique, etc. Mais si, pour reprendre le point de vue de Marcel Fournier ${ }^{23}$, le volontarisme «selon lequel il suffit d'avoir la ligne juste pour vaincre» ne nous éclairait pas vraiment? Et si le Parti n'avait pas trahi mais plutôt échoué?

\section{Les causes de l'échec du PCC}

En avril 1957, la revue Clarté du PCC publiait le texte d'un club montréalais du POP en plein coeur de la crise idéologique profonde qui secoua le Parti après les révélations du rapport Krouchtchev sur Staline. Ce document disait ceci:
Il n'y a cependant pas de doute que notre développement a été freiné par notre tendance à idéaliser et à dogmatiser le marxisme au lieu de l'enrichir et de l'approfondir en y greffant nos propres observations et expériences actuelles dans la lutte pour le socia- lisme au Canada. Notre style ennuyeux et dépourvu d'imagination (...) un style rempli du jargon de 1848 et de 1917, bourré du ver- biage soviétique contemporain; notre approche froide et «poli- tique» des gens; nos prophéties incessantes sur le désastre écono- mique qui nous guette, prophéties que nous répétons presque avec une satisfaction méchante (...) notre admiration et appui incondi- tionnels, sans aucun sens critique, de l'Union soviétique; toutes ces pratiques nous ont éloignés de nos frères canadiens. ${ }^{24}$

Cette analyse met l'accent sur les erreurs comme facteur d'explication. Elle suppose que ces «erreurs» auraient pu être corrigées, que la «trahison» aurait pu être évitée et surtout que la révolution aurait pu s'accomplir au Canada si la classe ouvrière avait eu de meilleurs dirigeants et un Parti plus ouvert et moins dogmatique. Mais il demeure qu'il n'y a pas eu de situation révolutionnaire au Canada au vingtième

\footnotetext{
22 Irving Abella, Nationalism, Communism and Canadian Labour (Toronto, University of Toronto Press, 1973), $256 \mathrm{p}$.

23 Marcel Fournier, Communisme et anticommunisme au Québec, 1920-1950 (Montréal, Éd. Albert Saint-Martin, 1979), 167 p.

24 «Statement to the National Committee from a Montreal club of the L.P.P.», Clarté (avril 1957): 6-14 (notre traduction).
} 
siècle. Pour maintenir la classe ouvrière dans un état de subordination politique et idéologique, la bourgeoisie canadienne n'a pas eu besoin de recourir au fascisme, par exemple, comme ce fut le cas dans certains pays européens où cela a favorisé la radicalisation des travailleurs. Ce que Marcel Fournier dit pour le Québec, à savoir qu' «aux principales phases de son histoire, le mouvement communiste (n'a) pas trouvé réuni (...) l'ensemble des conditions favorables à son développement» ${ }^{25}$ est bien entendu aussi vrai pour le Canada.

Il faut donc regarder du côté des conditions objectives pour comprendre l'échec du Parti. C'est ainsi qu'il nous apparaît clair que le type de développement économique que l'on a connu au Canada et la proximité des Etats-Unis, la composition de la classe ouvrière canadienne, les divisions ethniques, la place du Canada dans les relations internationales, le système fédéral lui-même avec les contraintes qu'il impose aux tiers partis qui veulent accéder au pouvoir et à une représentation équitable au Parlement, bref, il nous apparaît clair que l'ensemble de ces facteurs objectifs ont empêché l'éclosion d'une situation révolutionnaire et l'émergence d'un PC représentatif et fort.

\section{L'historien(ne) et le parti communiste}

Abordons maintenant les rapports de l'historien(ne) à un objet aussi particulier qu'un parti communiste. Nous l'avons vu avec l'histoire «officielle», avec les analyses des «erreurs», des «succès» et des «échecs» du Parti: il n'y a pas d'histoire plus controversée que celle-là. Il y a là deux niveaux de problèmes que nous ne pourrons présenter que brièvement: a) faire l'histoire du PC en partant du marxisme; b) faire l'histoire du PC comme de tout autre objet et les problèmes spécifiques qu'elle pose.

Pour aller à l'essentiel, nous dirons que si le marxisme continue d'offrir une des seules bases d'explication vraiment satisfaisante pour comprendre l'histoire de l'humanité, la pratique concrète du matérialisme historique s'est vu imposer par le stalinisme un style, une méthode et une approche théorique que l'on pourrait résumer par l'expression «adopter l'esprit de parti en histoire». Staline et la III ${ }^{\mathrm{e}}$ Internationale des partis communistes ont ainsi hypothéqué la recherche historique ${ }^{26}$ en l'assujettissant aux aléas des controverses politiques. En vertu de cette approche, l'historien(ne) marxiste était confiné à un corridor épistémologique marqué par les quatre postulats suivants: 1- l'histoire a un sens, c'est celui qui mène inéluctablement au communisme, à la société sans classes; 2- la classe ouvrière est porteuse de la mission historique qui consiste à faire la révolution pour accélérer le changement histo-

\footnotetext{
25 Marcel Fournier, op. cit., 127.

26 Comme l'a montré Georges Haupt dans L'historien et le mouvement social (Paris, Maspéro, 1980), 1 à 40 .
} 
rique vers cette société sans classes; 3 - la classe ouvrière est une, tous ses membres ont les mêmes intérêts; 4- il en découle la nécessité d'un parti unique, d'avant-garde, guide de la révolution.

En conséquence, il faut placer l'histoire du parti (porteur du sens historique au sens plein du terme) sous la responsabilité de la direction du Parti, ce qui entraîne une officialisation du travail des historien(ne)s et d'inévitables limites à la recherche. C'est ainsi qu'on voit surgir des questions prenant la forme de véritables tabous, comme l'histoire officielle du PCC en témoigne abondamment: ainsi, Tim Buck, qui dirigea le Parti de 1929 à 1964, doit absolument avoir participé à la fondation du Parti car cela lui donne une auréole de légitimité à laquelle aucun autre dirigeant ne peut prétendre; on ne doit pas parler de la dissolution de l'Internationale communiste, ou de telle défaite du Parti, ou encore du congrès de 1947 de l'aile québécoise du Parti qui fut marqué par l'expulsion de la majorité des membres francophones du PCC. Et bien entendu, à travers tout cela, il y a l'apologie constante du rôle du Parti, auquel on doit rester fidèle malgré ses erreurs car il représente rien de moins que l'Histoire en marche vers le Progrès.

Or, aujourd'hui, même les historiens du Parti communiste français reconnaissent qu'il faut désacraliser l'histoire des partis communistes, la sortir des commissions du Comité central ou du Bureau politique, en soumettre les résultats à la critique des non-communistes et ouvrir les archives aux chercheurs. On reconnaît même, dans les travaux des Cahiers de l'Institut Maurice Thorez ${ }^{27}$, qu'il n'y a pas de théorie marxiste toute prête d'avance pour expliquer la signature du pacte germanosoviétique par exemple... Il faudra donc forger de nouveaux concepts, développer de nouvelles approches, dans un climat de recherche et d'échange plus productif et ne pas hésiter à remettre en cause les postulats mềmes d'un «marxisme-léninisme» ossifié.

Par ailleurs, faire l'histoire du PC, est-ce faire l'histoire d'un parti comme les autres ou faire l'histoire $d u$ parti de la classe ouvrière? Nous croyons qu'il faille répondre non à ces deux questions. Le PC n'est certes pas un parti ordinaire, ne serait-ce qu'à cause du projet global qu'il véhicule et qui en fait pratiquement un mouvement social autant qu'un parti politique. Mais on ne voit pas non plus en vertu de quel apriorisme l'historien(ne) devrait lui concéder le titre de parti de la classe ouvrière, surtout lorsque la classe ouvrière, qui a parfois la tête bien dure, ne se rallie pas, électoralement ou autrement, au PC.

Faire l'histoire d'un parti communiste, c'est d'abord et avant tout faire l'histoire d'un mouvement social. Cela doit conditionner toute approche d'un PC et de son histoire. Car cela implique que le parti

\footnotetext{
27 Voir en particulier les articles de J. Burles, S. Wolikow et C. Willard dans le numéro spécial des Cahiers de l'Institut Maurice Thorez, «Le P.C.F. et l'Histoire», 29-30 (1979).
} 
communiste s'inscrit dans une formation sociale selon un mode qui lui est propre et qui est radicalement différent des autres partis. On n'a qu'à penser à l'attitude des PC devant le phénomène électoral, à l'importance qu'ils accordent à l'éducation de leurs militants, aux types de rapports qu'ils entretiennent avec la presse de parti, les syndicats et les organisations populaires et enfin aux références incessantes au modèle marxiste et à l'expérience soviétique pour mesurer l'ampleur des différences entre un PC et un autre parti politique.

En conclusion, faire l'histoire d'un parti communiste c'est également chercher à comprendre comment se réalise l'adéquation du parti à la société globale dans une conjoncture donnée. Ce qui implique des recherches sur la composition sociale de son membership et de son électorat, l'étude de son discours et de ses pratiques, et, bien entendu, l'étude de ses rapports avec les partis ou groupements extra-nationaux comme les Internationales, les comités de liaison, etc... C'est aussi situer leur praxis par rapport à une doctrine, d'une part, et par rapport aussi à ce point de référence essentiel aux yeux de tout communiste et de tout historien(ne): la classe ouvrière elle-même et son évolution, sa différenciation en couches sociales et ethniques, sa conscience donnée et sa praxis réelle.

À ce stade-ci des recherches, doit-on opposer une histoire militante à une histoire scientifique? Une histoire-bataille, politiquement engagée, à une histoire quantitative, sociologique? Nous croyons qu'il $\mathrm{y}$ a là un faux problème. Si un $\mathrm{PC}$ est une sorte de miroir déformant du mouvement ouvrier, il faut certes étudier le miroir et le processus de déformation mais il faut aussi connaître ce qui est reflété dans l'image, en l'occurrence le mouvement ouvrier dans son ensemble. Nous hésitons beaucoup à suivre Jean Chesneaux ${ }^{28}$ qui s'en prend à l'histoire scientifique en lui opposant justement les vertus de l'histoire militante. Jacques Julliard n'a-t-il pas raison de signaler que «donner un statut scientifique à l'histoire ouvrière constituait bel et bien une forme de contribution militante au mouvement ouvrier» ${ }^{29}$ ?

Pour sa part, Georges Haupt a cherché à opposer à l'histoire traditionnelle une «histoire ouvrière résolument sociale» ${ }^{30}$, ce qui ne l'empêchait pas d'étudier des institutions politiques comme la $\mathrm{II}^{\mathrm{e}}$ et la $\mathrm{III}^{\mathrm{e}}$ Internationale! Peut-on vraiment exclure l'histoire des luttes, des orga-

\footnotetext{
28 Jean Chesneaux, Du passé faisons table rase? (Paris, Maspéro, 1976).

29 Jacques Julliard, intervention à la table ronde «Histoire ouvrière, histoire sociale», Le mouvement social, 100 (1977): 51.

30 Georges Haupt, op. cit.
} 
nisations, des hommes et des femmes? Après tout, le politique révèle le social et il en exprime les contradictions. Encore faut-il en éviter les pièges et les hypothèques théoriques et politiques, éviter d'isoler le politique et développer collectivement l'histoire globale de la classe ouvrière canadienne, seul pôle de référence significatif face aux Partis qui prétendent véhiculer le sens de son histoire. 